\title{
Zigbee Communication on Machine to Machine Smart Home Micro Grid for Efficient Controlling System
}

\author{
Purwanto $^{1}$, Hermawan ${ }^{2}$, Suherman $^{3}$ \\ \{purwanto@live.undip.ac.id ${ }^{1}$ \} \\ Universitas Diponegoro, Indonesia ${ }^{1,2,3}$
}

\begin{abstract}
Data communication between sensors and actuator devices with users in the energy monitoring and control system on the smart grid is very dependent on the existence of a gateway device. This causes the control process to be ineffective because it is very dependent on the Gateway as the communication center. For that we need an effective approach so that the communication process can take place only on the relevant device, i.e. using M2M technology. In its development, the use of M2M technology has challenges related to congestion, error communication and delay so that further research is needed. In this research the development of a smart home micro grid will use M2M technology which enables communication between devices in the smart grid area. One component needed in M2M communication is Zigbee which is a medium for data communication without wires. Zigbee performance is measured using RSSI and Throughput with scenarios without or with obstructions as well as scenarios through the selection of router configurations. Experimental results show optimal performance of zigbee with scenarios in obstacle areas at a distance of 35 meters and in areas without barriers at a distance of 60 meters. In the router configuration selection scenario, it can be concluded that direct configuration is the optimal choice rather than using a router. For this reason, when using a configuration through a router, the number of sending nodes must be minimized.
\end{abstract}

Keyword: Smart Home Micro Grid, Machine To Machine, Zigbee Communication, Green Environment.

\section{Introduction}

Sufficient and adequate energy supply becomes a global problem, given the increasing energy demand and the depletion of raw materials for energy generation. In 2008, world energy demand was 474 exo joules and $85 \%$ of the world's electricity came from burning fossil materials (oil, coal and natural gas). In Indonesia, energy usage has reached 157,992 GWh, and most of it comes from fossil fuels (coal and petroleum). Several strategic steps have been taken, namely by developing energy management systems [1][2] and Non-intrusive Load Monitoring (NILM) systems to monitor and control the use of electricity [3]. The next step is to use non-fossil / renewable energy (solar cells, wind, water, etc.) instead of fossil fuels. The development and use of renewable energy are becoming increasingly important. Especially with the issue of increasingly globalized $\mathrm{CO} 2$ emissions that are contrary to the preservation of the global environment

Development and utilization of renewable energy sources require a process that was not short and also the support of fossil energy sources still very necessary so that integration 
between energy sources must be developed. The presence of Smart grids is a solution to integrate alternative energy sources (nuclear, geothermal) and renewable (wind, water, air, solar, etc.) with the ability to increase efficiency, reliability and security through automatic control and the use of modern communication technology [1]. Smart grids will certainly reduce dependence on the use of fossil fuels so as to reduce pollution and operational costs. The integration of energy sources allows the distribution of generation stations which not only occur on the provider side but also on consumers.

To support better control of smart grid devices, data communication between grid devices is needed through the development of a Machine to Machine (M2M) communication model. M2M does not only provide machine to man communication, but rather machine to machine communication. M2M technology enables device control processes to be more effective because it is not centered on the Gateway but between devices that are involved in a control process will be able to directly communicate. Utilization of M2M technology has been used in previous studies [4][5][6][7][8] for energy management systems in smart home or building, smart grids, smart meters and others. In this research, the development of smart home microgrid will use M2M technology which enables communication between devices in the smart grid area.

This paper discussed the designing of smart home micro grid using M2M technology that allows communication between devices in the smart home area. The organization of the paper was as follow: First, background and existing methods. Related works were described in Sec. 2. Smart home micro grid was describing in Sec. 3. Experiment and result was discussing in Sec. 4. Conclusions were describing in Sec. 5.

\section{Researchs Method}

Utilization of M2M technology has been used in previous studies [4][5][6][7][8] for energy management systems in smart home / building, smart grids, smart meters and others. Researchers [4] developed M2M with Bluetooth as a communication media on home area networks (HAN) to support data communication in gathering information related to the energy used. Researchers [5] developed M2M communication from sensor devices on cloud energy management systems. Researchers [6] developed M2M communication in the process of controlling and transferring measured energy data. Researchers [7] developed M2M communication networks on smart home and researchers [8] developed M2M communication networks on smart street lighting.

M2M technology development has several problems related to delay, congestion and communication errors. Niyato et. al. [9] proposed an approach to overcome problems in M2M network design by optimizing traffic concentration using the Dynamic Programming algorithm so that the optimal number of nodes for each concentrator can be determined. Zubair [10] proposed a method to improve zigbee performance by combining intelligence on smart meters and M2M devices. However, at the Gateway level, several problems still arise, including those related to congestion, delay and communication errors. This study proposes a direct communication model between devices so as to reduce problems that have arisen before. 


\section{Smart Home Micro Grid}

The existence of houses that still rely on conventional power plants that are sourced from fossil fuels can be possible to be integrated with non-fossil power plants that utilize renewable energy, for example solar power plants. Smart home equipped with appliances and home area network can be upgraded to form a grid for energy supply. Smart micro grid is a grid for energy supply in the home area that allows integration of the two energy sources. Through this micro grid, there will be automatic coordination and control in the use of energy supplies. Micro grid response occurs when the sensor detects that the energy supply from the solar panel is in optimal condition so that the electricity supply switches to that source.

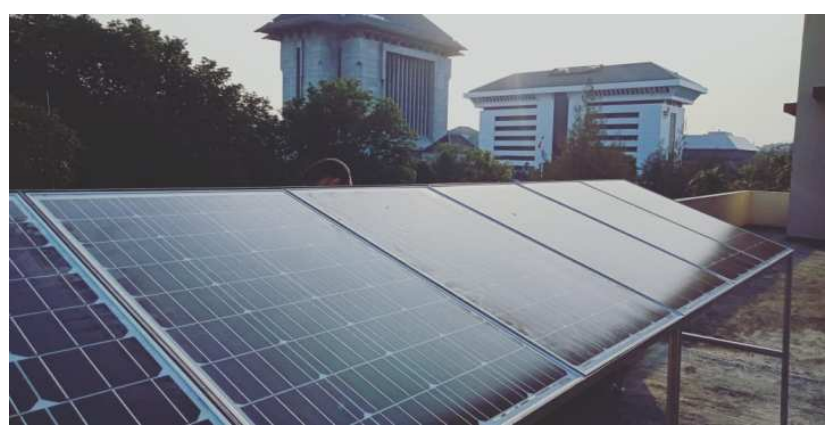

Fig. 1. Solar Panel Installation.

\subsection{Micro Grid Architecture}

Indonesia has begun to implement smart grids in home areas that are integrated with the utility network. Most of the renewable energy sources used comes from solar energy that is adapted to the tropical climate. In previous studies, a solar panel installation has been carried out which is integrated with the Utility network, as as shown in the Figure 2. Electricity derived from solar energy is very helpful in supporting home electricity supply, so it is not dependent on utility and ultimately has an impact on the decline in utility electricity bills.

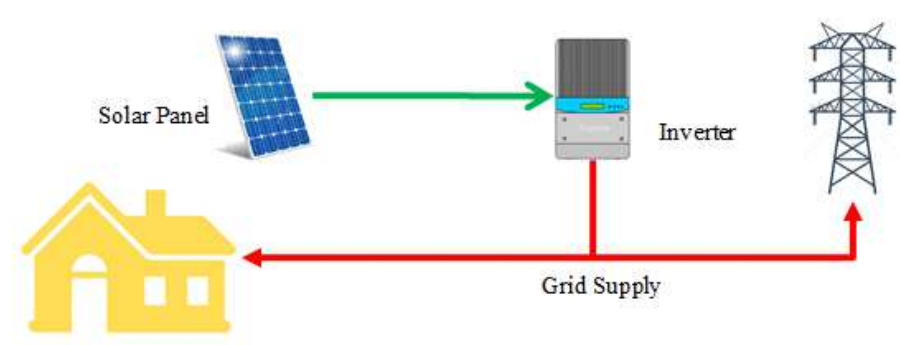

Fig. 2. Smart Home Microgrid Architecture.

Figure 2 shows the microgrid architecture used for the home area. In this architecture, the electricity network from renewable energy integrated with the utility network supplies 
electricity needs in the home area. The number of solar panels used is adjusted to the electricity needs of the home electrical appliances. The process of converting solar energy into electricity is carried out by solar panels in the form of direct current electricity (DC). For the needs of electrical equipment with alternating current (AC) an inverter is needed so that the electricity from the solar panels can be used by the load. To coordinate the electricity supply process on this smart grid, an energy management system was developed so that it can monitor and control devices on the smart grid. This smart grid is also equipped with a smart meter to get electrical data. Besides that, it also has other sensors to support the energy management process. Electrical data obtained, then analyzed for monitoring and controlling processes on smart grid devices.

\subsection{Machine to Machine Communication}

M2M communication usually resides between embedded devices through wired and wireless communication network media without user intervention. There are several types of M2M communication architectures as shown in Figure 3 below, namely: several MTCs communicate with MTC servers that are in the operator domain through APIs (a). The next type is the MTC server is outside the operator domain and is not controlled by operator (b). And the last type is the MTC device communicates with other MTC devices without going through an intermediary MTC server (c).

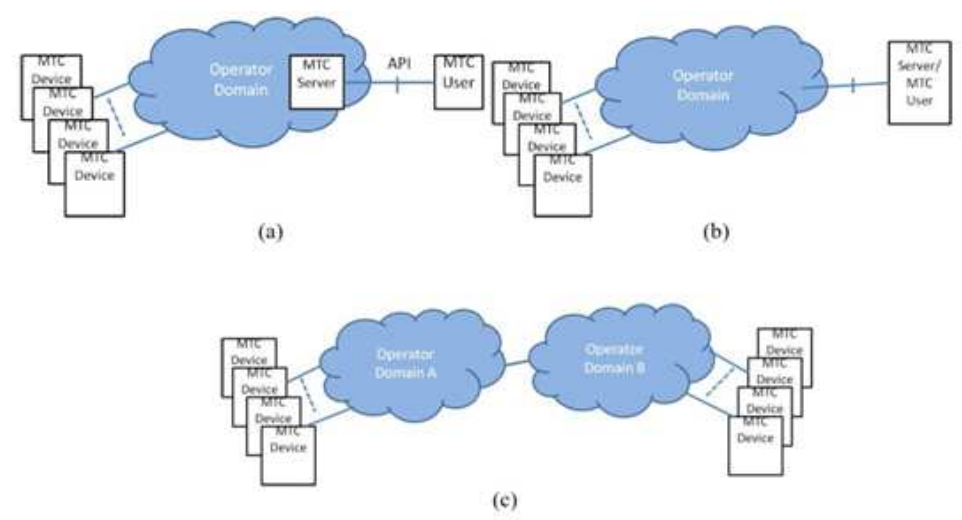

Fig. 3. Machine to Machine Architecture.

The ETSI M2M architecture consists of three domains: Device Domain, Network Domain and Application Domain as shown in Figure. 4. M2M equipment without a Service Capability Layer Device (DSCL) can be connected to a Network Domain through an M2M gateway, which has a standard Service Capability Gateway (GSCL) layer. M2M Network Domain connects M2M devices/gateways to M2M applications through a standard Network Service Capability (NSCL). SCL exposes M2M functionality through a series of open interfaces. Both functions and interfaces are defined in the ETSI specifications. 


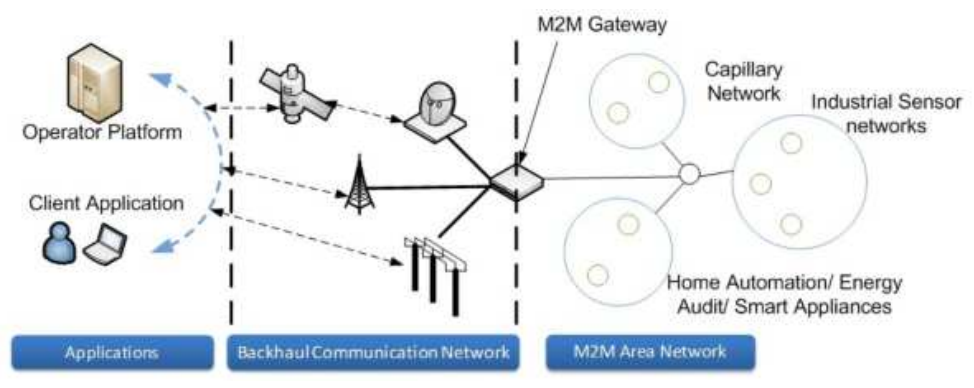

Fig. 4. Hirarki level ETSI Architecture.

\section{Result and Discussion}

The results of experiments conducted in the form of micro grid devices using solar panels and smart meters accompanied by $\mathrm{M} 2 \mathrm{M}$ communication. Solar panels installed in the area of the house are placed on the roof of the house which has the potential to get a match and maximum sunlight. 6 solar panels were installed and each panel produced DC electricity of $100 \mathrm{wp} / 12 \mathrm{~V}$. To store DC electricity generated, the smart grid is equipped with 6 batteries with a capacity of $100 \mathrm{Ah}$ each. Smart meter was used to retrieve data in the form of voltage and current signals in real time. This smart meter was mounted on a cable that connects the solar panel and the battery to measure the voltage and current entering the battery. Besides that, it was also installed on the home electricity panel to measure the voltage and current from the electricity grid. The following picture on Figure 5 is a smart meter installation. Zigbee communication performance testing is measured using the Received Signal Strength Indicator (RSSI) to get the minimum reception power level so that it can communicate optimally. The minimum power level received according to the ITU-T standard is $-83 \mathrm{dBm}$. Beyond this power, the zigbee receives data with a large delay.

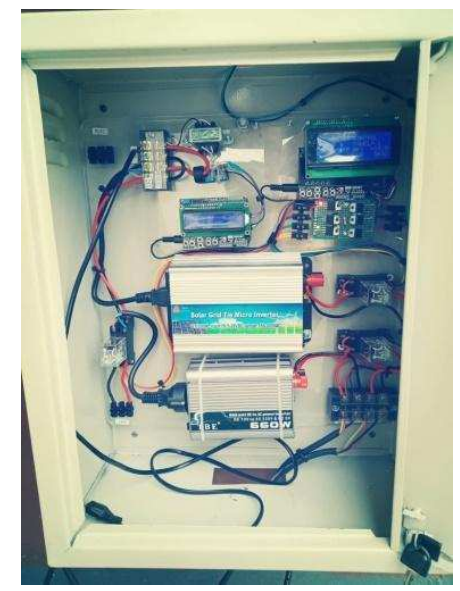

Fig. 4. Smart meter with embedded zigbee M2M. 
To measure RSSI, the transmitter power $\left(P_{t}\right)$ and receiver power $\left(P_{r}\right)$ are calculated first as follows:

Where,

$$
P_{r}=P_{t} \times G_{t} \times G_{r}\left(\frac{\lambda}{4 \pi d}\right)^{2}
$$

$$
\begin{aligned}
G_{t} & =\text { Transmitter Gain } \\
G_{r} & =\text { Receiver Gain. } \\
\lambda & =\text { wavelength and distance between sender and receiver. }
\end{aligned}
$$

RSSI can be formulated as:

$$
R S S I=10 \log \log \frac{P_{r}}{P_{R e f}}
$$

In the experiments conducted, the optimal distance that can be applied on the barrier areas in each zigbee is about 35 meters with a signal power of $-83 \mathrm{dBm}$ and an average traffic delay of $0 \mathrm{~ms}$. Signal power will be reduced to $-95 \mathrm{dBm}$ when the distance is extended to 50 meters and the average traffic delay to $1200 \mathrm{~ms}$. In areas without barriers, the optimal distance for each zigbee is around 60 meters with a signal power of $-80 \mathrm{dBm}$ and an average traffic delay of $0 \mathrm{~ms}$. Signal power will be reduced to $-95 \mathrm{dBm}$ when the distance is extended to 85 meters and the average traffic delay becomes $1200 \mathrm{~ms}$.

Zigbee performance is also measured based on Throughput (TP). This measure will provide information on the amount of data sent and received in a certain time period. Besides that, the amount of time delay during sending and receiving data packets is measured. To calculate throughput, use the following formula:

$$
T P=\frac{8 \times \text { number of bytes }}{\text { total transmission time }(\mathrm{sec})}
$$

Throughput measurements are performed on the topology used on the M2M network with the zigbee protocol. The choice of this topology will affect the size of the output generated. There are two scenarios in this measurement, namely by using a point to point topology between the coordinator and the end device without going through a router device. Then the second scenario is to use a configuration in a wider area using a router. The measurement results in each scenario can be shown in Figures 5 and 6. In the router configuration selection scenario, it can be concluded that direct configuration is the optimal choice rather than using a router. For this reason, when using a configuration through a router, the number of sending nodes must be minimized. 


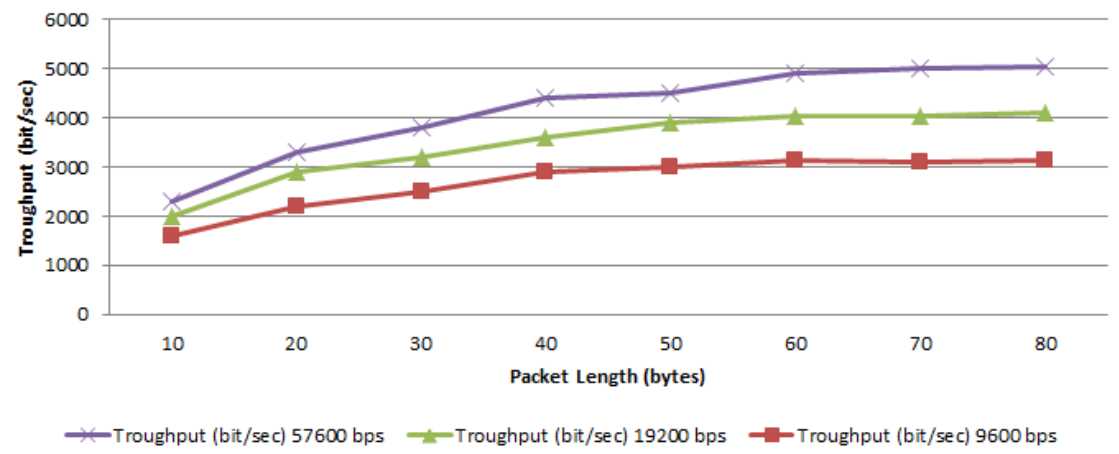

Fig. 5. Throughput measurement in point to point configuration

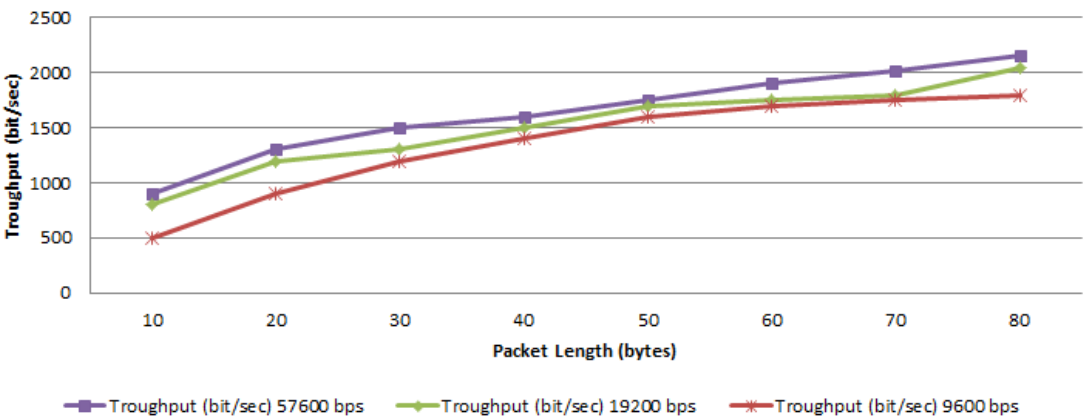

Fig. 6. Throughput measurement in not direct configuration using a router

\section{Conclusion}

The development of a smart home micro grid using M2M technology enables communication between devices in the smart grid area to be more optimal. This M2M communication uses zigbee protocol to provide data communication network between sensor devices, monitor devices and control devices. The use of zigbee as a communication protocol has been evaluated by measuring RSSI and Throughput through several scenarios. In the experiments conducted, the optimal distance that can be applied to the barrier areas in each zigbee is about 35 meters and in areas without barriers, the optimal distance for each zigbee is around 60 meters with each signal power of $-83 \mathrm{dBm}$ and $-80 \mathrm{dBm}$. In the router configuration selection scenario, it can be concluded that the direct configuration is the optimal choice rather than using a router. For this reason, when using a configuration through a router, the number of sending nodes must be minimized.

\section{References}

[1] N. Iksan, S. H. Supangkat, and I. G. B. B. Nugraha, "Home Energy Management System: A framework Through Context Awareness," in International Conference on ICT for Smart Society, 2013, pp. 1-4. 
[2] N. Iksan, E. D. Udayanti, A. Arfriandi, and D. A. Widodo, "Automatic Control using Fuzzy Techniques for Energy Management on Smart Building," in 2018 International Conference on Computer Engineering, Network and Intelligent Multimedia (CENIM), 2018, pp. 156-160.

[3] N. Iksan, J. Sembiring, N. Hariyanto, and S. H. Supangkat, "Residential Load Event Detection in NILM Using Robust Cepstrum Smoothing Based Method.," Int. J. Electr. Comput. Eng., vol. 9, no. 2, 2019.

[4] N. Sahu and V. Dehalwar, "Intelligent Machine to Machine Hommunication in Home Area Network for Smart Grid," in 2012 Third International Conference on Computing, Communication and Networking Technologies (ICCCNT'12), 2012, pp. 1-6.

[5] G. Suciu, O. Fratu, L. Necula, A. Pasat, and V. Suciu, "Machine-To-Machine Communications for Cloud-Based Energy Management Systems within SMEs," in 2016 IEEE 22nd International Symposium for Design and Technology in Electronic Packaging (SIITME), 2016, pp. 114-117.

[6] K. Y. Kamel, A. S. Ibrahim, A. Zekry, and M. Abouelatta, "On Design of M2M Smart Energy Control and Management Architecture Using Smart Metering Technology," in 2016 11th International Conference on Computer Engineering \& Systems (ICCES), 2016, pp. 452-460.

[7] Z. Wang and X. Xu, "Smart Home M2M Networks Architecture," in 2013 IEEE 9th International Conference on Mobile Ad-hoc and Sensor Networks, 2013, pp. 294-299.

[8] D. A. Widodo, N. Iksan, and A. F. Suni, "Design of Embedded Zigbee Machine to Machine Smart Street Lighting System," in 2017 2nd International conferences on Information Technology, Information Systems and Electrical Engineering (ICITISEE), 2017, pp. 392-395.

[9] D. Niyato, L. Xiao, and P. Wang, "Machine-To-Machine Communications for Home Energy Management System in Smart Grid," IEEE Commun. Mag., vol. 49, no. 4, pp. 53-59, 2011.

[10] Z. M. Fadlullah, M. M. Fouda, N. Kato, A. Takeuchi, N. Iwasaki, and Y. Nozaki, "Toward Intelligent Machine-to-Machine Communications in Smart Grid," IEEE Commun. Mag., vol. 49 , no. 4, pp. 60-65, 2011. 